\title{
Existence and stability of intrinsic localized modes in a finite FPU chain placed in three-dimensional space
}

\author{
M. Kimura ${ }^{\dagger}$, A. Mitani, S. Doi \\ †kimura.masayuki.8c@kyoto-u.ac.jp
}

Department of Electrical Engineering, Kyoto University, Katsura, Nishikyo-ku, Kyoto 615-8510, Japan

Fermi-Pasta-Ulam (FPU) chain is well known model in which energy localized vibrations exist. Such energy localized vibrations are called intrinsic localized modes (ILMs) or discrete breathers (DBs). This paper discusses existence and stability of ILMs in a finite Fermi-Pasta-Ulam chain which is placed in three-dimensional space, namely, motion of each mass is not constraint to the axis of the chain. First we derive dispersion relations of longitudinal and transversal waves. It is shown that the dispersion relations are changed with respect to the initial stretch or compression of the chain. By using Newton-Raphson method, three kinds of ILM, namely, longitudinal, transversal, and rotating modes, are found in the chain. All masses moves along the axis of the chain in longitudinal modes. The relationship between the frequency and the amplitude distribution completely coincides with ILMs in the traditional FPU chain in which motions of masses are constraint along the axis. On the other hand, main oscillations of transversal modes are perpendicular to the axis. In rotating modes, all masses rotate around the axis. Distribution of the radius of rotations are localized. Stability analysis reveals that almost all the longitudinal and the transversal ILMs are unstable. However, we found a narrow parameter region of the initial stretch in which the transversal ILM becomes stable.

Keywords: intrinsic localized mode, discrete breather, FPU chain, transversal mode, rotating mode.

\section{Introduction}

Intrinsic localized mode (ILM), which is also called discrete breather (DB), is spatially localized and temporally periodic solution in nonlinear lattices. Many researches, which have been reported since the latter half of the 20th century, show evidences of the existence of ILM in real physical systems [1]. In particular, observations of ILM in micro-structures, such as optic wave guides [2], Josephson junction ladders $[3,4]$, and micro-mechanical cantilever arrays [5], strongly suggest that ILM/DB can be utilized for applications in micro-/nano-technologies.

Recently, existence of ILM/DB has been investigated in low-dimensional carbon materials, namely carbon nanotubes [6], graphene [7, 8], graphane [9], etc. In addition to those two-dimensional carbon materials, an extremely thin carbon wire, namely a carbon monoatomic chain, was experimentally created [10]. The numerical observations of ILM/DB in two-dimensional carbon materials imply the existence of ILM/DB in the carbon monoatomic chain. The carbon monoatomic chain attracts researcher's attentions because of its characteristics in heat and electric conductance $[11,12]$. The aim of our research is to investigate whether ILM/DB exists in a carbon monoatomic chain or not and how ILM/DB affects the characteristics of the chain such as heat and electric conductance.

In carbon monoatomic chain, carbon atoms are arranged in one-dimension and they are placed in three-dimensional space. Therefore, the atoms can move not only along the axis of the chain but also perpendicular to the axis. Such systems are already investigated for curved biopolymer chains [13], polymer chain with secondary structure [14], and polyethylene chains [15]. A simpler model which is a straight Fermi-Pasta-Ulam (FPU) chain is treated in this paper for investigating an effect of extension of the dimension of space in which the chain is placed.

\section{FPU chain in $3 D$ space}

Fermi-Pasta-Ulam chain is originally proposed for investigating the equipartition of kinetic energy in nonlinear crystals [16]. Usually, motions of masses are constrained along the axis of the chain. However, if the chain is located in two- or three-dimensional space, the masses can move transversally as well as longitudinally (see Fig.1). The equation of motion of the masses which are allowed to move transversally is described as:

$$
\begin{aligned}
& m \ddot{\boldsymbol{r}}_{n}=\frac{\boldsymbol{d}_{n}}{\left\|\boldsymbol{d}_{n}\right\|}\left\{-\alpha\left(\left\|\boldsymbol{d}_{n}\right\|-\boldsymbol{l}_{0}\right)-\beta\left(\left\|\boldsymbol{d}_{n}\right\|-\boldsymbol{l}_{0}\right)^{3}\right\}- \\
& -\frac{\boldsymbol{d}_{n+1}}{\left\|\boldsymbol{d}_{n+1}\right\|}\left\{-\alpha\left(\left\|\boldsymbol{d}_{n+1}\right\|-\boldsymbol{l}_{0}\right)-\beta\left(\left\|\boldsymbol{d}_{n+1}\right\|-\boldsymbol{l}_{0}\right)^{3}\right\},
\end{aligned}
$$

where $\boldsymbol{r}_{n}$ is the three dimensional position vector from equilibrium position to each mass. $\boldsymbol{d}_{n}$ is the distance vector between $n$-th and (n-1)-th masses and defined as $\boldsymbol{d}_{n}=\boldsymbol{l}_{n}+\boldsymbol{r}_{n}-\boldsymbol{r}_{n-1}$. The nonlinear spring connecting neighboring masses has an equilibrium length of $l_{0}$. It is assumed that each mass is arranged along $x$-axis of Cartesian coordinate system and the entire chain is initially stretched or 
compressed. Therefore, initial positions of masses are denoted by $l_{n}=\left(l_{0}, 0,0\right)+\left(l_{1}, 0,0\right)$, where $l_{1}$ represents the initial stretch/ compression if it is positive/negative. In this paper, $m, \alpha$, and $l_{0}$ are set at unity, and the coefficient of the cubic nonlinear restoring force is fixed at $\beta=25$. The number of masses is chosen to be eight. The boundary condition is assumed to be periodic, namely, $d_{0}=d_{8}, d_{9}=d_{1}$.

The dispersion relation of Eq.(1) is obtained as follows:

$$
\begin{aligned}
& \omega_{x}=2 \sqrt{1+3 \beta l_{1}^{2}} \sin \left|\frac{k_{x}}{2}\right|, \\
& \omega_{y}=2 \sqrt{\frac{l_{1}+\beta l_{1}^{3}}{1+l_{1}}} \sin \left|\frac{k_{y}}{2}\right|,
\end{aligned}
$$

whrere $\omega_{x}$ and $\omega_{y}$ denote angular frequency of longitudinal and transversal waves, respectively. Since the chain is cylindrically symmetric, $\omega_{z}$ is the same as $\omega_{y}$. Eqs.(2) and (3) shows that there are upper bound for the frequencies $\omega_{x}$ and $\omega_{y}$ when the wave numbers $k_{x}$ and $k_{y}$ become $\pi$ (or $-\pi$ ), respectively. To exist an ILM in Eq.(1), the ILM should avoid to resonate with linear plain waves[1], namely, the frequency of the ILM $\Omega$ should be greater than $\omega_{x, \max }=2 \sqrt{1+3 \beta l_{1}^{2}}$ and/or $\omega_{y, \max }=2 \sqrt{\left(l_{1}+\beta l_{1}^{3}\right) /\left(1+l_{1}\right)}$.

\section{Intrinsic localized modes}

In the FPU chain placed in three-dimensional space, three kinds of ILM possibly exist, namely, longitudinal, transversal, and rotating ILMs. The longitudinal ILM that each mass moves along the axis of the chain as shown in Fig.2(a). The longitudinal ILM coincides with that in the FPU chain in which the motion of each mass is constrained along the axis. Therefore, the relationship between the frequency and the amplitude should be the same between them. However, the stability should be different because an effect of a perturbation perpendicular to the axis should be considered, which will be discussed in the next section.

Figure 3 shows the wave form of two kinds of longitudinal ILM. In Fig.3(a), two neighboring masses, namely, the 4th and 5th, oscillate in antiphase with the same amplitude while the other masses show very small amplitude oscillations. This type of ILM can be called Page mode [17]. In this paper, the longitudinal Page mode is abbreviated as L-P mode. Fig.3(b) shows a different ILM from L-P mode in symmetry. The 4th mass has the largest amplitude and the 3rd and 5th masses have the same amplitude which is rather smaller than that of the 4th mass but sufficiently large comparing with the other masses. This is called Sievers-Takeno mode [17]. Hence, the longitudinal Sievers-Takeno mode is abbreviated as L-ST mode. For both L-P and L-ST modes, all the $y$ - and $z$-components are always zero, since there is no perpendicular force as long as all the masses moves along the $x$-axis. Therefore, L-P and L-ST modes exactly the same as P and ST modes in the traditional FPU chain.

Transversal ILM in which each mass mainly vibrates perpendicular to the axis (see Fig.2(b)) is a specific ILM in the FPU chain placed in three-dimensional space. The

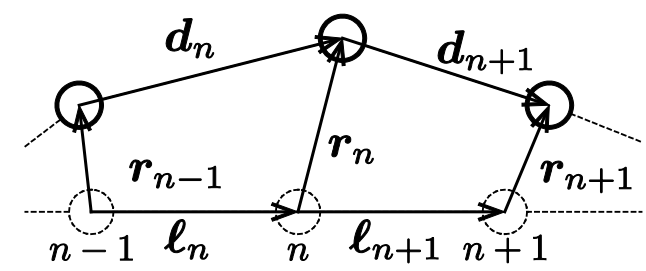

Fig. 1. Configuration of FPU chain.

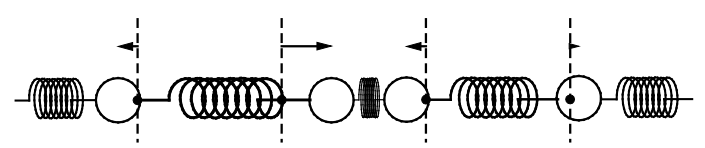

(a) Longitudinal mode

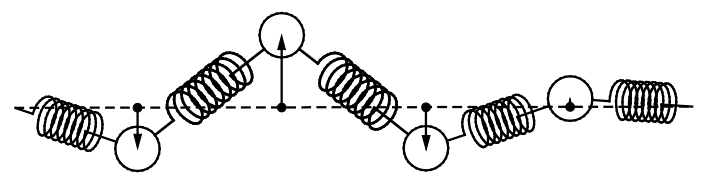

(b) Transversal mode

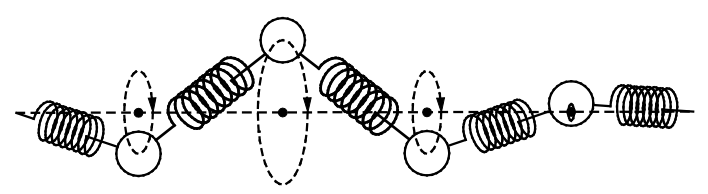

(c) Rotating mode

Fig. 2. Three kinds of ILM in FPU chain.
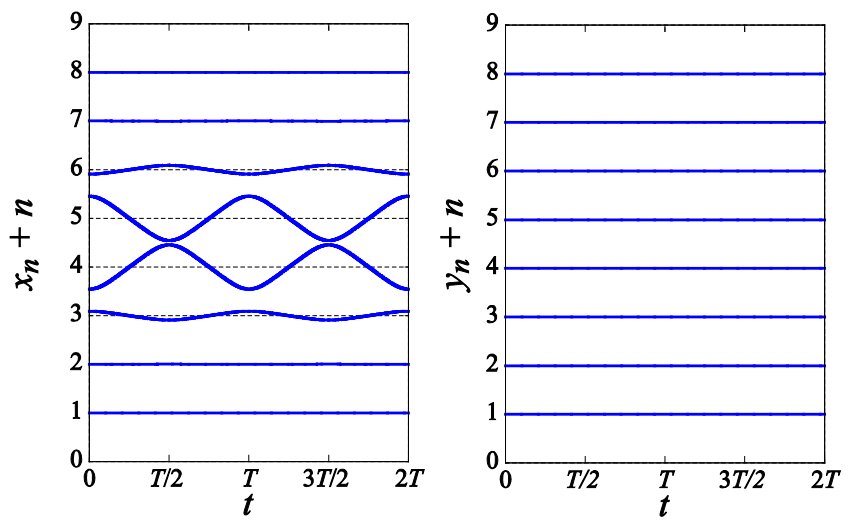

(a) Longitudinal P mode
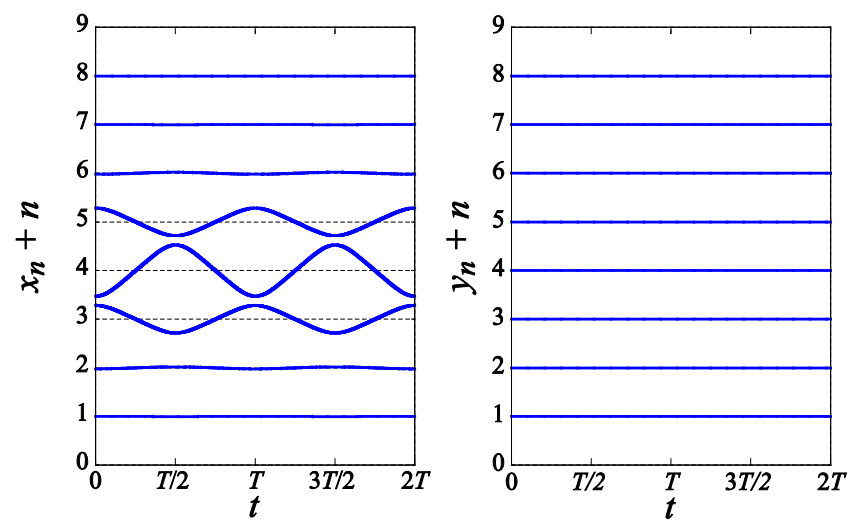

(b) Longitudinal ST mode

Fig. 3. Wave profile of longitudinal modes. $T=1, l_{1}=0$. The dotted line indicates the equilibrium position. Note that the solution is obtained by using the Newton-Raphson method for periodic solution. Thus, there is no energy radiation from the ILM core. 
motions of masses are shown in Fig.4. For the transversal ILMs, there exist two kinds of ILM as well as the longitudinal ILM. They are called transversal Page (T-P) mode and transversal Sievers-Takeno (T-ST) mode, which are shown in Fig.4(a) and 2(b), respectively. For both ILMs, each mass mainly oscillate along $y$-direction instead of $x$-direction. As shown in right panels, the amplitude distribution is clearly localized. Interestingly, each mass except 4th and 8th masses in Fig.4(b) has an initial shift in $x$-direction even though $l_{1}$ is set at zero. It implies that the chain is compressed toward the center of ILM. In addition, each mass oscillates along $x$-axis with a frequency twice as much as that of the motions along $y$-axis. The reason why the motion in $x$-direction is caused is that a displacement perpendicular to the axis of the chain causes a restoring force not only in the perpendicular direction but also in the longitudinal direction. The restoring force in the longitudinal direction does not depend on the sign of the perpendicular displacement. Therefore, the frequency of the longitudinal force is twice as much as that of the perpendicular force. It implies that the frequency of transversal ILM $\boldsymbol{\Omega}_{\mathrm{TILM}}$ has to satisfy both $\boldsymbol{\Omega}_{\mathrm{TILM}}>\omega_{x, \max } / 2$ and $\boldsymbol{\Omega}_{\text {TILM }}>\omega_{y, \max }$ simultaneously.

Rotational ILM is also found as shown in Fig.5. The 4th and 5th masses oscillate with large amplitude. The phase difference between $y_{n}$ and $z_{n}$ equals $\pi / 2$. Thus, all the masses rotate around the $x$-axis. In this case, the direction of rotation is the same among all of the masses. The phase difference between neighboring masses is $\pi$. Therefore, the amplitude distribution corresponds to that of Page mode. Then it can be classified into rotating Page mode. Rotating SieversTakeno mode should exist as well as the longitudinal and the transversal ILMs. However, we could not find it so far. It remains for future research.

\section{Stability analysis}

Generally, Page mode is not unstable in FPU chain because all of the characteristic (Floquet) multipliers are located on the unit circle in the complex plane [17]. On the other hand, Sievers-Takeno mode is unstable since one of the characteristic multiplier is located outside the unit circle [17]. This result is also valid for the system of Eq.(1) in which all the masses are constrained along the $x$-axis and the parameters are chosen as $m=1, \alpha=1, \beta=25, l_{0}=1$, $l_{1}=0$. All the characteristic multipliers are located on the unit circle as shown in Fig.6(a). However, if there is no constraint for masses, both the longitudinal P and ST modes are unstable even though the amplitude distribution and the wave form is completely the same as in the system having the constraint. In Fig.6(b), several characteristic multipliers are found inside and outside the unit circle, while the position of the other multipliers located on the unit circle is the same as in Fig.6(a). It implies that the longitudinal P mode becomes unstable because each mass is not constrained along the chain axis. The unstableness comes from the fact that there is a moment at which the chain is compressed, for instance, the spring between 4 th and 5 th masses are compressed around $t=T / 2,3 T / 2$. The compression will cause the buckling of the chain. In fact eigenvectors of characteristic multipliers located outside the unit circle have non-zero values in $y$ or $z$-components as shown in Fig.6(c). This means that the longitudinal ILM is unstable along the transversal direction.

The transversal ILMs are also unstable for almost all parameter region of the initial stretch $l_{1}$. However, narrow regions where all the characteristic multipliers are on the unit circle, namely, the absolute value of them equals unity, are found as shown in Fig.7. The regions exist for $l_{1}>0.5$. The mechanism is not unclear so far, but an initial stretch tends to stabilize the transversal ILMs.
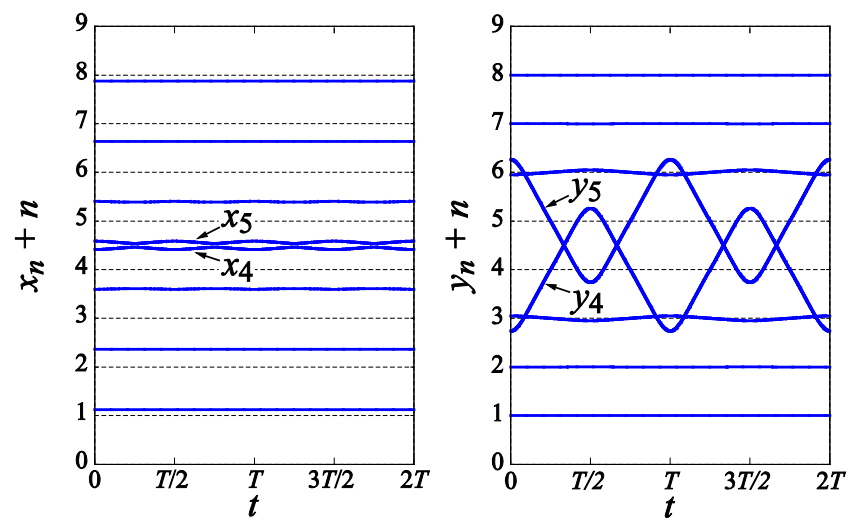

(a) Transversal P mode
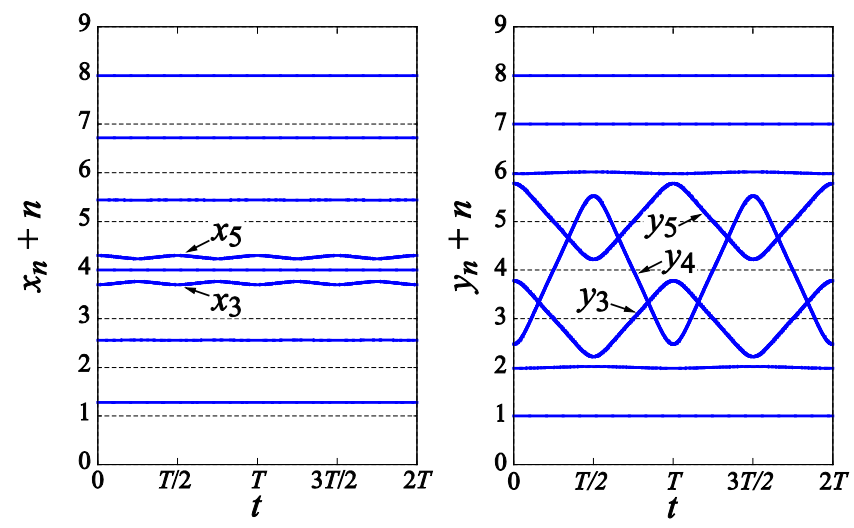

(b) Transversal ST mode

Fig. 4. Wave profile of transversal modes. $T=1, l_{1}=0$.
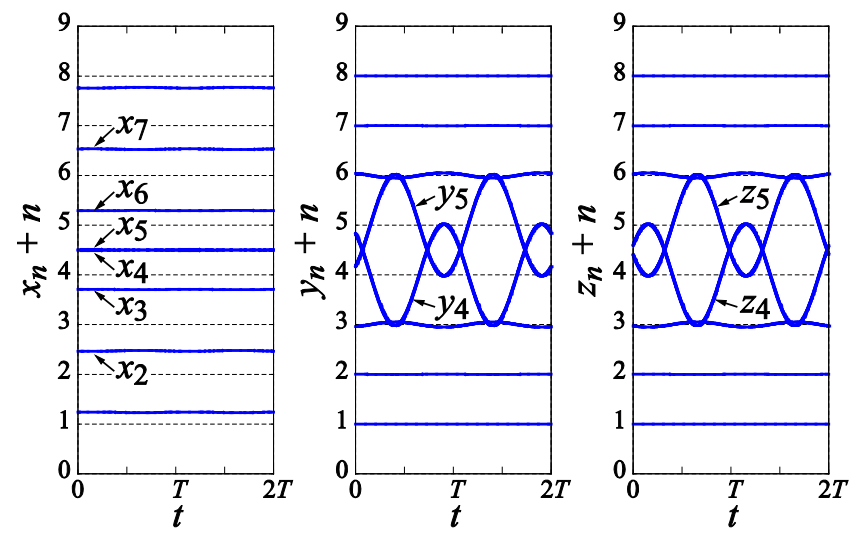

Fig. 5. Wave profile of rotating mode. $T=1.14, l_{1}=0.1$. The initial shift along the $x$-axis is also observed as well as the transversal ILM. 


\section{Conclusion}

In this paper, new kinds of ILM have been researched for an FPU chain placed in three-dimensional space. By using Newton-Raphson method, we successfully found the transversal ILMs and the rotating ILMs as well as the traditional longitudinal ILMs. Stability analysis has revealed that almost all the ILMs in the FPU chain is unstable even though the longitudinal ST mode is stable in the traditional FPU chain in which all the masses are constraint along the chain axis. According to the eigenvectors of unstable characteristic multipliers, it is strongly suggested that the buckling effect makes ILMs unstable. It implies that the buckling effect should be taken into account for studies on ILM in one- or two-dimensional systems in two- or

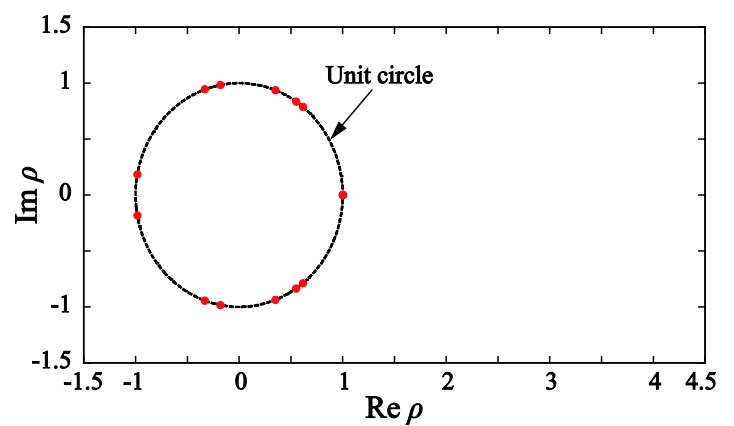

(a) Characteristic multipliers of the traditional P mode.

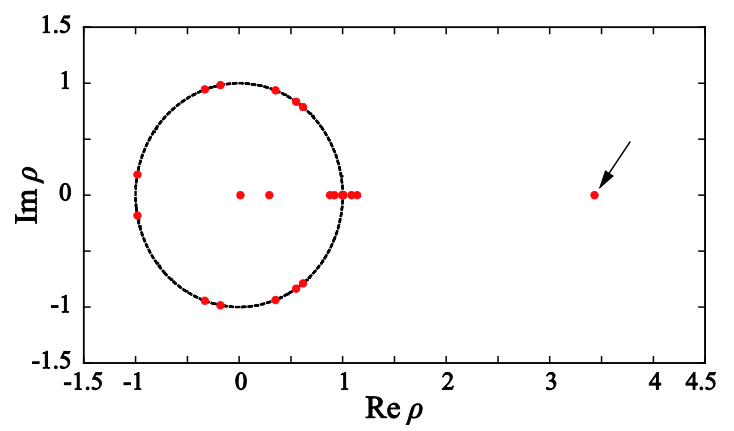

(b) Characteristic multipliers of the longitudinal P mode.

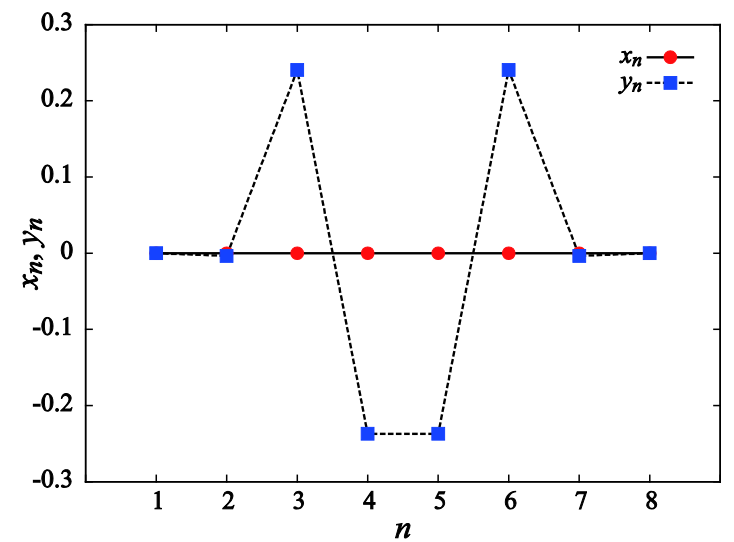

(c) Eigenvector of the characteristic multiplier indicated by arrow in (b).

Fig. 6. Characteristic multipliers of P mode in (a) the traditional FPU chain (b) the FPU chain located in three-dimensional space. (c) $x$ and $y$-components of an eigenvector of the characteristic multiplier indicated by the arrow in (b). Note that the velocity components of $x$-axis are also zero. three-dimensional spaces, respectively, such as carbon monoatomic chains, graphene sheets, carbon nanotubes, etc. We will discuss ILM in such real nano systems in the future.

Acknowledgements. One of the authors (MK) would like to thank Prof. Sergey V. Dmitriev, RAS, Russia and Prof. Y. Doi, Osaka University, Japan for fruitful discussion. This work was supported by the Ministry of Education, Culture, Sports, Science and Technology in Japan, Grant-in-Aid for Young Scientists (B) No.25820164.

\section{References}

1. S. Flach, A.V. Gorbach. Phys. Rep. 467, 1 (2008).

2. H. S. Eisenberg and Y. Silberberg and R. Morandotti and A. R. Boyd and J. S. Aitchison. Phys. Rev. Lett. 81, 3383 (1998).

3. P. Binder, D. Abraimov, A. V. Ustinov, S. Flach, Y. Zolotaryuk. Phys. Rev. Lett. 84, 745 (2000).

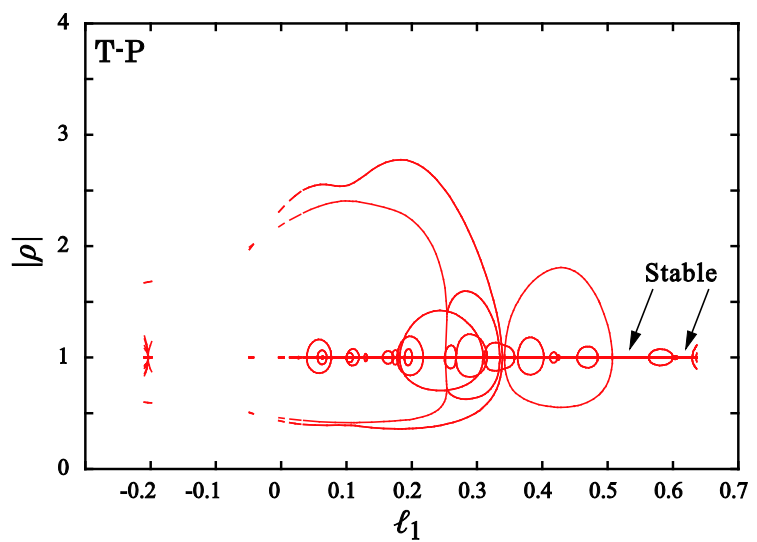

(a) Transversal P mode

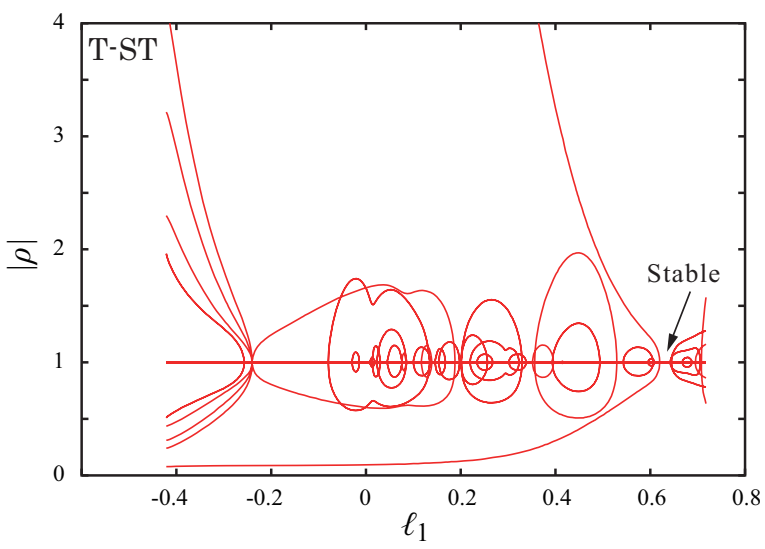

(b) Transversal ST mode

Fig. 7. Absolute value of the characteristic multipliers of transversal modes. The period of ILM is fixed at unity. The broken curves appeared in (a) for $l_{1}<0$ do not indicate that there is no solution. In the initially compressed chain, there is a moment at which the neighboring masses become very close each other. Since Eq.(1) has the distance at the denominator, the small distance increases the numerical error. Only the solutions which were successfully converged are shown in the figure. 
4. E. Trías, J. J. Mazo, T. P. Orlando. Phys. Rev. Lett. 84, 741 (2000).

5. M. Sato, B. E. Hubbard, A. J. Sievers, B. Ilic, D. A. Czaplewski, H. G. Craighead. Phys. Rev. Lett. 90, 044102 (2003).

6. Y. Kinoshita, Y. Yamayose, Y. Doi, A. Nakatani, T. Kitamura. Phys. Rev. B 77, 024307 (2008).

7. Y. Yamayose, Y. Kinoshita, Y. Doi, A. Nakatani, T. Kitamura. Europhys. Lett. 80, 40008 (2007).

8. Y. Doi, A. Nakatani. Journal of Solid Mechanics and Materials Engineering 6, 71 (2012).

9. G. M. Chechin, S. V. Dmitriev, I. P. Lobzenko, D. S. Ryabov. Phys. Rev. B 90, 045432 (2014).

10. C. Jin, H. Lan, L. Peng, K. Suenaga, S. Iijima. Phys. Rev.
Lett. 102, 205501(2009)

11. N. Wei, G. Wu, J. Dong. Phys. Lett. A 325, 403 (2004).

12. X. Chen, C. Ming, F.-X. Meng, J.-T. Li, J. Zhuang, X.-J. Ning. J. Appl. Phys. 114, 154309 (2013).

13. M. Ibañes, J. M. Sancho, G. P. Tsironis. Phys. Rev. E 65, 041902 (2002).

14. A.V. Zolotaryuk, P.L. Christiansen, A.V. Savin. Phys. Rev. E 54, 3881 (1996).

15. A.V. Savin, L.I. Manevitch. Phys. Rev. B 67, 144302 (2003)

16. E. Fermi, J. Pasta, S. Ulam. Studies of Non Linear Problems. The collected papers of Enrico Fermi Vol.2. University of Chicago Press. (1955) p. 978.

17. S. Flach, A. Gorbach. Chaos 15, 15112 (2005). 